\title{
A Novel Composite Based on Straw: PVAC/RSS
}

\author{
Chuanbao $\mathrm{Wu}^{1, \mathrm{a}}$ and Guoguang Xiao, ${ }^{1, \mathrm{~b}}$ \\ ${ }^{1}$ College of Chemistry and environmental engineering, Jiujiang \\ University, Qianjin Eath Road No.551, Jiujiang city, Jiangxi Province, \\ People's Republic of China \\ awucbao@163.com, ${ }^{b}$ libaiguoguang@163.com
}

\begin{abstract}
In order to overcome moisture absorption of straw based composite with poly(vinyl alcohol) as adhesive material, poly(vinyl acetate) (PVAC) was introduced into the preparation of composite based on rice straw slice (RSS). The effects of PVAC content, hot-pressing temperature and hot-pressing time on the performances were studied. Results showed that tensile strength of composite increased with the increases of PVAC content and hot-pressing temperature, and decreased with the increase of hot-pressing time. The tensile strength of composite arrived at 8.34 MPa when PVAC content was 50\%, hot-pressing temperature was $170{ }^{\circ} \mathrm{C}$ and hot-pressing time was $14 \mathrm{~min}$. Tensile elongation of composite increased with increasing PVAC amount. When PVAC contents were $17 \%$ and $50 \%$, tensile elongations were $13.5 \%$ and $20.9 \%$ respectively. The increasing rate was $54.9 \%$. Tensile elongation was higher than elongation at maximum tensile force. This indicated that PVAC/RSS composite had certain toughness.

Keywords: Environmentally friendly material, Composite, Hot pressing, Straw.
\end{abstract}

\section{Introduction}

Development of environmentally friendly materials is an important study direction of current and future material science field ${ }^{[1-3]}$. Environmentally friendly 
composites are more favorable to keep body health, decrease the consumption of energy and eliminate environmental pollution. Plant straw of wide source is an easily obtained raw material of producing environmentally friendly materials ${ }^{[4-6]}$. Composites with plant straw as main raw materials can be used in the manufacturing of general products. Plant straw includes many kinds, for example rice straw, corn straw and wheat straw etc. All these plant straws can be used to make composite. Rice is a widely planted food crop. So developing composite based on rice straw is of higher practical significance. Performance of plant straw based composites is determined to a large extent adhesive material. Poly(vinyl alcohol) is a long used adhesive material in our team because it has high structural similarity and compatibility. The composite with poly(vinyl alcohol) as adhesive material has high tensile strength and toughness. However this kind of composite also has a shortcoming: moisture absorption. In order to solve this problem, the application of PVAC in the straw based composite was studied. PVAC has ester groups, which was expected to not only have certain compatibility with straw but also allow composite with low moisture absorption.

\section{Materials and Methods}

Materials. PVAC with molecular weight of 30000-50000 was purchased from Guoyao Chemical Reagent company (Shanghai, China) and was used as received. Rice straw was obtained from farmland of Jiujiang's suburb and was dried in the sun before use. When rice straw was used, it was chopped into about $1.5 \mathrm{~cm}$ segments and then was sheared into slice and filament. After removing the powder produced during shearing with a 20 mesh sieve, RSS was obtained. Water was from urban water-supplying system. Rapespeed oil was common cooking oil and was used as received.

Preparation of PVAC/RSS Composite. 25 g RSS was weighed into beaker and then $10 \mathrm{~mL}$ water was added, followed by stirring for $5 \mathrm{~min}$ so that the RSS was uniformly moistened. Thereafter, different amounts of PVAC were weighed and added into moistened RSS, followed by stirring for 5 min. The mixed raw 
material was put in a $125 \mathrm{~mm} \times 125 \mathrm{~mm} \times 2 \mathrm{~mm}$ iron mold and then was hot-pressed on MZ-3012 machine at 10MPa pressure. After cooling to room temperature, the product was taken out to be tested.

Measurement of Composite. $2.0 \mathrm{~cm}$ wide strips were cut out from products to test tensile performance. Tensile strength and tensile elongation were measured on MZ-2000D.D1 electronic universal testing machine (Mingzhu test machine company, Jiangdu, Jiangsu, China) with tensile speed as $5 \mathrm{~mm} / \mathrm{min}$. Square with side length as $4 \mathrm{~cm}$ was selected from products and its four vertices were selected as hardness-measurement points. The four vertices were labeled respectively as point 1, 2, 3 and 4 according to hardness-increasing order. Shore hardness was measured with LX-A Shore A type durometer (Mingzhu test machine company, Jiangdu, Jiangsu, China).

\section{Results and Discussion}

The Effect of PVAC Amount on the Strength of Composites. A series of PVAC/RSS composites were prepared at $170{ }^{\circ} \mathrm{C}$ by changing the amount of PVAC from $5 \mathrm{~g}$ to $25 \mathrm{~g}$. The changes of tensile strength and strength at tensile fracture with increasing PVAC amount were drawn in Fig. 1. It can be seen that two kinds of strength increases with the increase of PVAC content. When PVAC content was 17\%, two kinds of strength were 3.3 MPa and 1.7 MPa respectively. At $50 \%$ of PVAC content, two kinds of strength arrived at maximum values. The tensile strength was 8.34 MPa and strength at tensile break was 6.36 MPa. The increasing rates of two kinds of strength were $153 \%$ and $269 \%$ respectively. In the composites, RSS could not bond together itself and PVAC played an adhesive role. With the increase of PVAC content, more and more bonding points of RSS formed and so the strength increased. From Fig. 1, it can also be seen that tensile strength is always higher than strength at tensile fracture at any PVAC content. This indicates that RSS experienced a yielding course after maximum tensile force. 

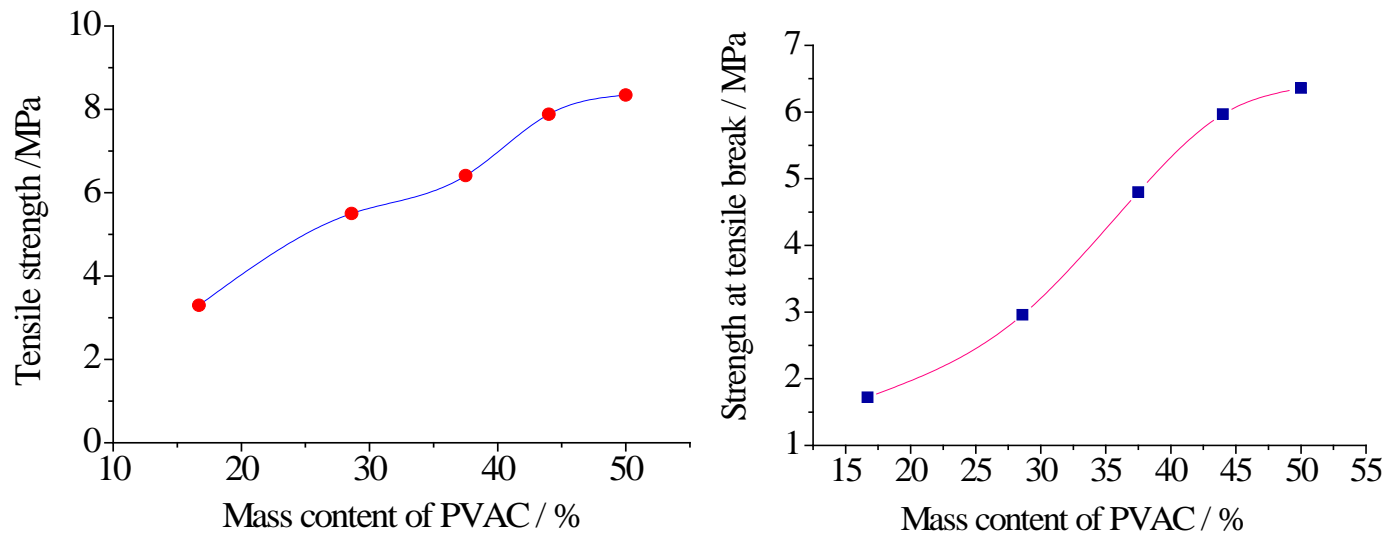

Fig. 1 The change of strength with increasing the amount of PVAC

The Effect of PVAC Amount on the Stretch of Composites. The changes of tensile elongation and elongation at tensile break with increasing PVAC amount were drawn in Fig. 2. It can be seen that as tensile strength two kinds of stretches also increased with increasing PVAC amount. When PVAC contents were 17\% and $50 \%$, tensile elongations were $13.5 \%$ and $20.9 \%$ respectively. The increasing rate was 54.9\%. When PVAC content increased from $17 \%$ to $50 \%$, the elongation at maximum tensile force increased from $11.1 \%$ to $15.9 \%$ and the increasing rate was $43.2 \%$. Elongation increasing with PVAC content was maybe related to the increase of bonding points. More bonding points made composites experience longer tensile time before fracture. From Fig. 2, it can also be seen that tensile elongation is always higher than elongation at maximum tensile force. This indicates that PVAC/RSS composite had certain toughness and this toughness had relation with both PVAC and RSS because both of two kinds of raw materials have certain toughness. 


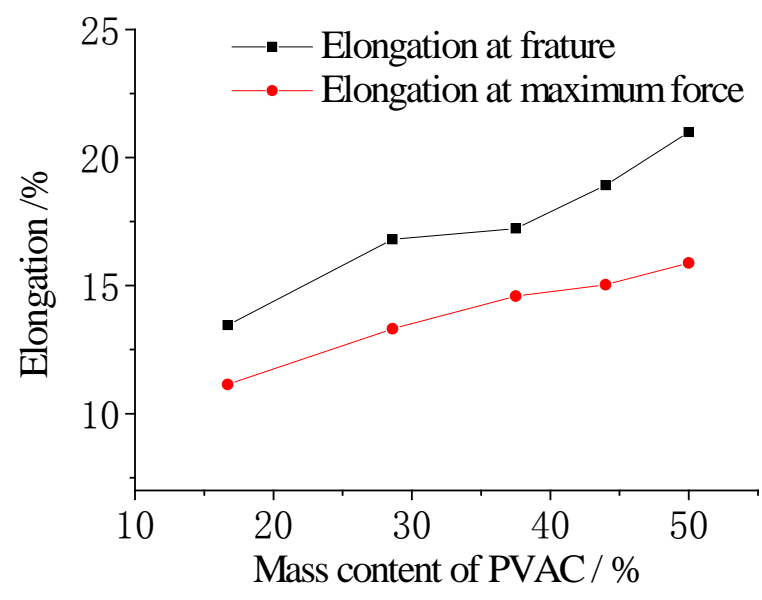

Fig. 2 The effect of PVAC content on elongation of composites

The Effect of Hot-pressing Temperature on the Performance of

Composites. A series of PVAC/RSS composites were prepared with $37.5 \%$ content of PVAC at different hot-pressing temperatures. The changes of tensile strength and tensile elongation with increasing hot-pressing temperature were drawn in Fig. 3. It can be seen that two tensile performances increase with the increase of hot-pressing temperature. When hot-pressing temperature changed from $140^{\circ} \mathrm{C}$ to $180^{\circ} \mathrm{C}$, tensile strength increased from 3.3 MPa to 8.3 MPa. The increasing rate arrived at $152 \%$. High hot-pressing temperature was favorable to disperse PVAC into RSS and increase bonding points. When hot-pressing temperature changed from $140^{\circ} \mathrm{C}$ to $180^{\circ} \mathrm{C}$, tensile elongation increased from $14.6 \%$ to $19.5 \%$. The increasing rate arrived at $33.6 \%$. This indicates that composite of high strength had long yielding time. 

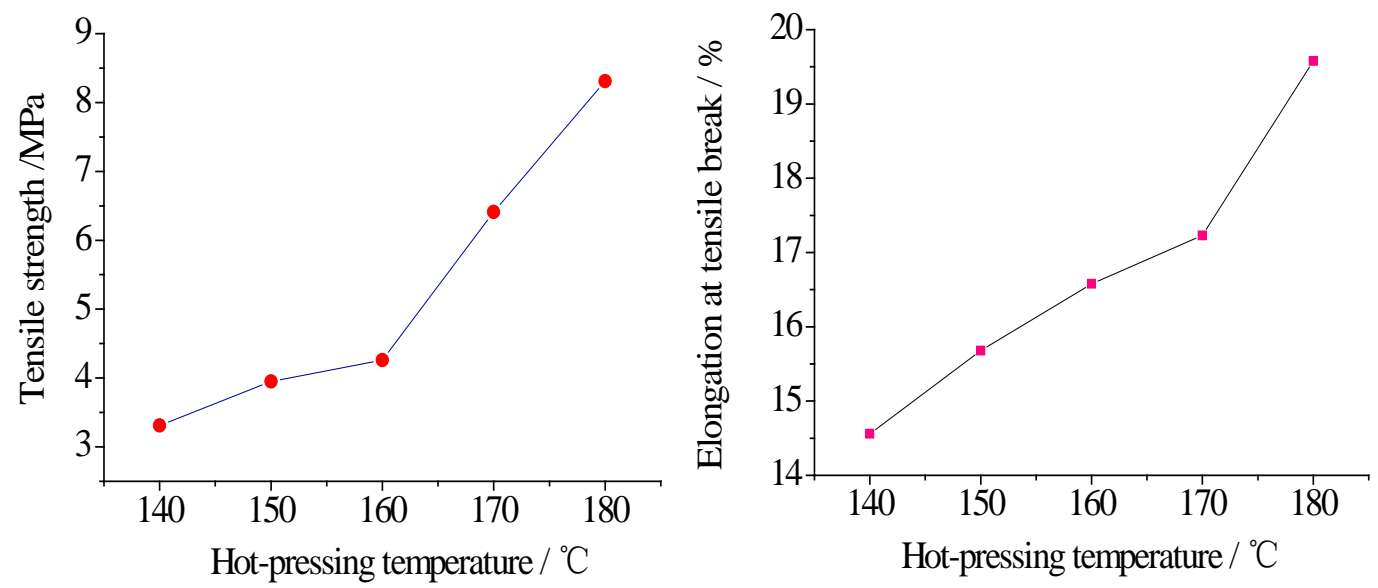

Fig. 3 The effect of hot-pressing temperature on the tensile performances of composites

The Effect of Hot-pressing Time on the Performance of Composites. A series of PVAC/RSS composites were prepared with 37.5\% content of PVAC at different hot-pressing times. The change of tensile strength with increasing hot-pressing time was drawn in Fig. 4. It can be seen that tensile strength decreases with the increase of hot-pressing time. When hot-pressing time changed from $10 \mathrm{~min}$ to $16 \mathrm{~min}$, tensile strength decreased from 6.77 $\mathrm{MPa}$ to 5.0 $\mathrm{MPa}$. On one hand, the increase of hot-pressing time was favorable to disperse PVAC into RSS. On the other hand, long heat treatment probably disrupted the structure of raw materials. 


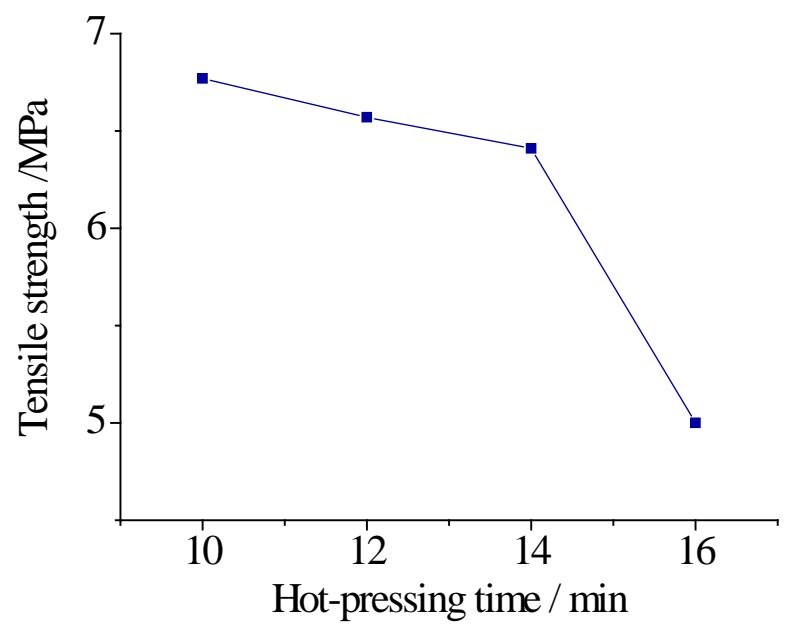

Fig. 4 The effect of hot-pressing time on tensile strength of composites

\section{Summary}

PVAC was a desirable adhesive material in the preparation of straw based composite. It has good compatibility with straw and strength. The tensile strength of PVAC/RSS composite approached the composite prepared with poly(vinyl alcohol). But its moisture absorption was not so high as poly(vinyl alcohol).

\section{Acknowledgements}

This work was financially supported by the Jiangxi Technology Supporting Program (20121BBE50008).

\section{References}

[1] T. Masui, T. Honda, Wendusu and N. Imanaka: Dyes Pigments Vol. 99 (2013), p. 636

[2] M. Forster: J. Cleaner Prod. Vol. 23 (2012), p. 195 
[3] L. Wang, J. Littlewood and R.J. Murphy: Renew. Sust. Energ. Rev. Vol. 28 (2013), p. 715

[4]X.J. Zhang, T.S. Zhaob, N. Harac, Y.Z. Jinc, C.Y. Zeng, Y.u Yoneyama and N. Tsubaki: Fuel Vol. 116(2014), p. 34

[5] L. Zhang and Y.C. Hu: Mater Design Vol. 55(2014), p. 19

[6] J.J. Liu, C.J. Jia and C.X. He: AASRI Procedia Vol. 3(2012), p. 89 